\title{
The Effect of Thrombin Concentration on Fibrin Clot Structure Imaged by Multiphoton Microscopy and Quantified by Fractal Analysis
}

\author{
R. M. Bateman, ${ }^{*}$ H. Leong, ${ }^{*}$ T. Podor, ${ }^{*}$ K. C. Hodgson, ${ }^{* *}$ T. Kareco, ${ }^{*}$ and K. R. Walley*
}

*Respiratory Medicine Division, University of British Columbia, The James Hogg iCAPTURE Centre, St.Paul's Hospital, British Columbia, Canada V6Z1Y6

**Pathology and Laboratory Medicine, University of British Columbia, The James Hogg iCAPTURE Centre, St.Paul’s Hospital, British Columbia, Canada V6Z1Y6

Fibrin clots are an important component of chronic cardiovascular thrombosis and are associated with cardiovascular dysfunction, stroke and formation of microthrombi in systemic inflammatory states. During coagulation, thrombin selectively cleaves fibrinogen resulting in the formation of linear fibrin polymers that form by end-to-end and side-to-side association. Here we present a novel assay for real-time imaging of fibrin polymerization using fluorescence laser scanning multiphoton microscopy (MPM) and fractal analysis for quantification of clot structure.

Human blood, collected in acid citrate dextrose tubes, was centrifuged (2x, for 8 minutes @ 500 x g) to produce platelet poor plasma (PPP). Fibrin polymerization was initiated at room temperature by addition of thrombin to PPP and imaged (Fig.1) through incorporation of fluorescently labeled fibrinogen (5\% vol/vol Oregon Green labeled fibrinogen to PPP fibrinogen) at 0,1,2,3,5,10 and 15 minutes. $900 \mathrm{~nm}$ fs pulses (Ti-Sapphire laser) were focused through a 63x/1.2 NA objective (Leica AOBS SP2 microscope system) to a clot depth of $20 \mu \mathrm{m}$. Fluorescent images were acquired at $400 \mathrm{~Hz} / 3$ frame averages, emission bandwidth 500-650nm. At high doses of thrombin (0.1 and 0.05 units $/ \mathrm{ml})$, clots formed within 1-3 minutes of initiation. At lower thrombin doses (0.025 and 0.01 units/ml), clots were unstable over the first 5 minutes (generating motion artifacts), but were stable ten minutes after initiation. To quantify clot structure, images were processed using MATLAB (The MathWorks, Inc., MA) and quantified by fractal analysis (TruSoft Int'l Inc., FL). The image processing algorithm consisted of background signal subtraction, median filter, image thresholding (Otsu's method [1]) and binarization. Fractal dimension was calculated using the box dimension estimation method. The box dimension, $\mathrm{Db}$, is defined as the exponent of $\mathrm{N}(\mathrm{d}) \sim 1 / \mathrm{d}^{\mathrm{Db}}$, where $\mathrm{N}(\mathrm{d})$ is the number of boxes of linear size (d) required to cover a set of points distributed in a 2D plane. As thrombin concentration increased the clot $\mathrm{Db}$ value rapidly increased then seemingly reached a plateau at a thrombin concentration of $0.1 \mathrm{unit} / \mathrm{ml}$, see Fig $2 \mathrm{~B}$, indicating that fibrin clot structure was dependent on thrombin concentration and stabilized at the highest thrombin concentration tested.

This MPM imaging technique of fibrin clots combined with fractal analysis of clot structure offers a rapid, reproducible and promising approach to investigate clotting mechanisms in disease states and to test the efficacy of drug treatments on clotting kinetics, clot structure and clot fibrinolysis.

References

[1] N. Otsu, IEEE 9(1) (1979) 62.

[2] This work was supported by Heart and Stroke Foundation Canada (HSFC), Michael Smith Foundation for Health Research (British Columbia) and CIHR/HSFC IMPACT Postdoctoral Fellowships. 

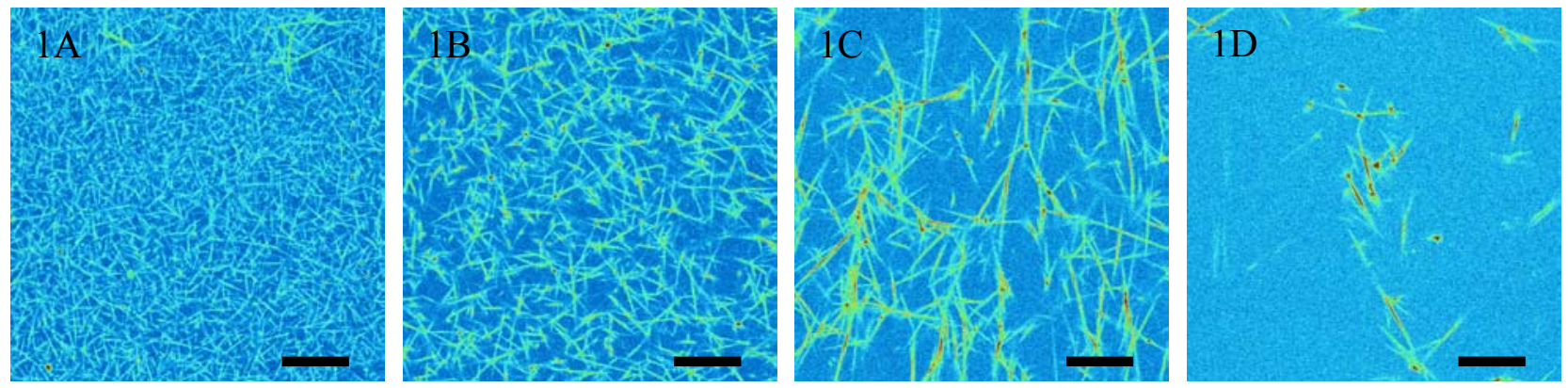

0

255

Fig.1. Fibrin clots imaged ten minutes after addition of thrombin (IIa) to platelet poor plasma. Clots were imaged at room temperature at a clot depth of $20 \mu \mathrm{m}$ by two photon excitation (900nm FWHM $12 \mathrm{~nm}$ ) of Oregon Green labeled fibrinogen (5\% - total fibrinogen content) which was incorporated into the fibrin clot during polymerization. Figs $1 \mathrm{~A}, 1 \mathrm{~B}, 1 \mathrm{C}$ and $1 \mathrm{D}$ were obtained after addition of $0.1,0.05,0.025$ and 0.01 units $/ \mathrm{ml}$ of thrombin, respectively. Scale bar $=20 \mu \mathrm{m}$
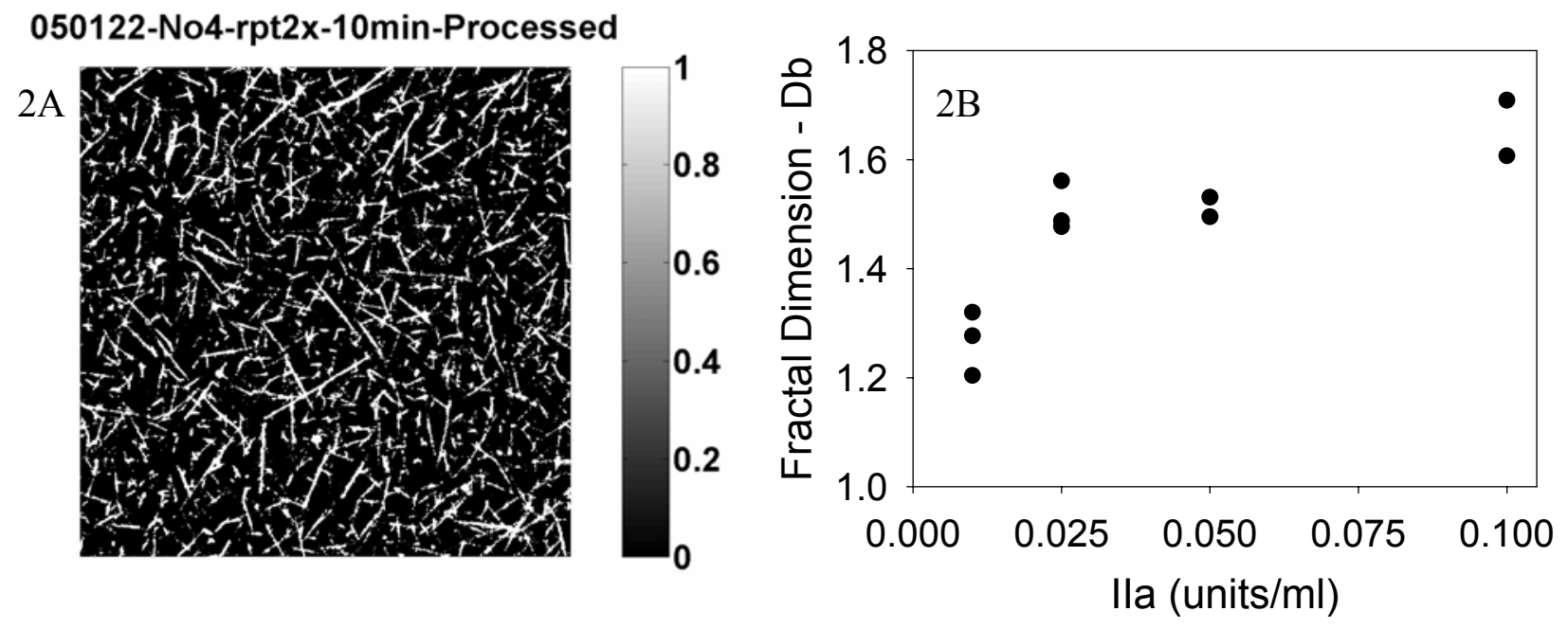

Fig.2. Fractal analysis of human fibrin clots. Clot images (Fig. 1) were processed using MATLAB prior to fractal analysis. Fig. 2A shows an example of a processed clot image. Fig. 2B shows the effect of thrombin (IIa) concentration on the fractal box dimension, $\mathrm{Db}$, of fibrin clots. 